\section{ORIGINAL RESEARCH}

A. Mahmood

G. Bibat

A.-L. Zhan

I. Izbudak

L. Farage

A. Horska

S. Mori

S. Naidu

\title{
White Matter Impairment in Rett Syndrome: Diffusion Tensor Imaging Study with Clinical Correlations
}

BACKGROUND AND PURPOSE: RTT, caused by mutations in the methyl CPG binding protein 2 (MeCP2) gene, is a disorder of neuronal maturation and connections. Our aim was to prospectively examine FA by DTI and correlate this with certain clinical features in patients with RTT.

MATERIALS AND METHODS: Thirty-two patients with RTT underwent neurologic assessments and DTI. Thirty-seven age-matched healthy female control subjects were studied for comparison. With use of a 1.5T MR imaging unit, DTI data were acquired, and FA was evaluated to investigate multiple regional tract-specific abnormalities in patients with RTT.

RESULTS: In RTT, significant reductions in FA were noted in the genu and splenium of the corpus callosum and external capsule, with regions of significant reductions in the cingulate, internal capsule, posterior thalamic radiation, and frontal white matter. In contrast, FA of visual pathways was similar to control subjects. FA in the superior longitudinal fasciculus, which is associated with speech, was equal to control subjects in patients with preserved speech (phrases and sentences) $(P=.542$ ), whereas FA was reduced in those patients who were nonverbal or speaking only single words $(P<.001)$. No correlations between FA values for tracts and clinical features such as seizures, gross or fine motor skills, and head circumference were identified.

CoNCLUSIONS: DTI, a noninvasive technique to assess white matter tract pathologic features, may add specificity to the assessment of RTT clinical severity that is presently based on the classification of $\mathrm{MeCP} 2$ gene mutation and $\mathrm{X}$-inactivation.

\begin{abstract}
ABBREVIATIONS: AIR = automated image registration; $\mathrm{ALIC}=$ anterior limb of internal capsule; $\mathrm{CC}=$ corpus callosum; CG1, CG3, CG4 = cingulate gyrus; $\mathrm{CG} 2$ = cingulum; $\mathrm{CP}=$ cerebral peduncle; $\mathrm{CPG}=$ cytosine guanosine dinucleotide; $\mathrm{DTI}=$ diffusion tensor imaging; $\mathrm{EC}=$ external capsule; FA = fractional anisotropy; FW = frontal white matter; FX/ST =fornix/stria terminalis; $\mathrm{GC}=$ genu of corpus callosum; ICBM = International Consortium of Brain Mapping; IFO = inferior fronto-occipital fasciculus; $\mathrm{LI}=$ laterality index; $\mathrm{MCP}=$ middle cerebellar peduncle; PCR = posterior corona radiata; PLIC = posterior limb of internal capsule; PTR = posterior thalamic radiation; $\mathrm{ROI}=$ region of interest; $\mathrm{RTT}=$ Rett syndrome; $\mathrm{SCC}=$ splenium of corpus callosum; SCP = superior cerebellar peduncle; SLF = superior longitudinal fasciculus; $\mathrm{SS}=$ sagittal stratum.
\end{abstract}

$\mathbf{R}$ TT, a neurodevelopmental disorder that predominantly affects female patients, is caused by mutations in the methyl CPG binding protein $2(\mathrm{MeCP} 2)$ gene located at Xq28. ${ }^{1,2}$ RTT occurs in 1 in 22,000 girls, and patients with RTT seem to develop normally up to 6 months of life. ${ }^{3,4}$ Nonetheless, deceleration in the velocity of head growth

Received February 19, 2009; accepted after revision June 18.

From the Department of Internal Medicine (A.M.), University of Texas Medical Branch, Galveston, Texas; Department of Neurogenetics (G.B., S.N.), Hugo Moser Research Institute, Kennedy Krieger Institute, Johns Hopkins University, Baltimore, Maryland; F.M. Kirby Research Center (S.M.), Kennedy Krieger Institute, Johns Hopkins University, Baltimore, Maryland; Russell H. Morgan Department of Radiology and Radiological Science (I.I., L.F., A.H., S.M.), Johns Hopkins University, Baltimore, Maryland; Medical School (L.F.), Brasilia University, Brasilia, DF, Brazil; and Department of Radiology (A.-L.Z.), Zhangzhou Hospital of Fujian Medical University, Zhangzhou, Fujian Province, China.

Supported by National Institute for Child Health and Human Development grant P01-HD24448; National Institute on Aging grant AG-20012; National Center for Research Resources grant RR-15241; and Johns Hopkins Institute for Clinical and Translational Research grant UL1RR025005.

Please address correspondence to Sakkubai Naidu, MD, 707 North Broadway, Baltimore, MD 21205; e-mail: naidu@kennedykrieger.org

Indicates open access to non-subscribers at www.ajnr.org

DOI 10.3174/ajnr.A1792 occurs as early as 4 months of life up to 2 years, leading to microcephaly in most patients. ${ }^{5}$ Patients lose the ability to use words and experience poor hand use with cognitive and motor deficits.

Pathologic studies show that brain weight in RTT is reduced compared with control subjects. ${ }^{6}$ This decrease in the weight of the brain is not generalized. It is more significant in the cerebral hemispheres and less prominent in the cerebellum. Brain volume measurements in vivo show regional alterations, with relative preservation in the posterior occipital and posterior temporal regions. ${ }^{7}$ The regional reduction in volumes is not attributed to atrophy ${ }^{8}$ but to poor neuronal maturation ${ }^{9,10}$ and connections. ${ }^{11}$ Increased cell-packing attenuation because of poor dendritic arborization has been attributed to poor neuronal interconnectivity resulting in microcephaly. ${ }^{6,11,12}$

Structure-specific pathologic features of white matter can be assessed noninvasively by DTI. ${ }^{13,14}$ Different quantitative measures can be derived from a DTI study. Our prospective study used the directionality of water diffusion, quantified by $\mathrm{FA},{ }^{15}$ to investigate the tract-specific abnormalities and their correlations with some clinical features in patients with RTT. 


\section{Materials and Methods}

\section{Subjects}

Thirty-two girls with RTT syndrome were included in the study based on $\mathrm{MeCP} 2$ gene mutation analysis and clinical parameters. They underwent neuroimaging and neurologic evaluation as part of the Natural History study in RTT being conducted at the Kennedy Krieger Institute. In-depth clinical assessments included neurologic status, head circumference, history of seizures, respiratory irregularities, gait, and speech. Imaging and clinical tests were performed during the same admission. Thirty-seven age-matched healthy female control subjects were included for comparison. There was no significant age difference between patients with RTT vs control subjects ( $5.5 \pm 0.7$ vs $6.1 \pm 0.7$ years, $P=.58$ ). All patients were sedated for MR imaging scan, and control subjects were not.

The Institutional Review Board at Johns Hopkins University approved the study, and informed consent was obtained from legal guardians.

\section{Data Acquisition}

A 1.5T MR unit (Philips Medical Systems, Best, the Netherlands) was used for data acquisition. Conventional MR imaging consisted of sagittal T1-weighted, axial T2 fast spin-echo, and axial fluid-attenuated inversion recovery images. Conventional brain MR images were obtained in the patients and control subjects. We acquired DTI data using a single-shot echo-planar imaging sequence with the sensitivity encoding, parallel-imaging scheme (reduction factor, 2.5). ${ }^{16}$ The FOV was $240 \times 240 \mathrm{~mm}$; $96 \times 96$ imaging matrix was zero-filled to $256 \times 256$ pixels. Transverse sections of $2.5 \mathrm{~mm}$ in thickness were acquired parallel to the anterior/posterior commissure line. DTI was encoded along 30 independent orientations, ${ }^{17}$ and the b-value was $700 \mathrm{~s} / \mathrm{mm}^{2}$. Three DTI datasets were acquired for each participant, and the acquisition time per dataset was approximately 6 minutes.

\section{Data Processing}

For DTI processing, software (DTIStudio) built in-house was used (H. Jiang, S. Mori; Johns Hopkins University and Kennedy Krieger Institute, Baltimore, Maryland; http://lbam.med.jhmi.edu). ${ }^{18} \mathrm{We}$ first realigned the images using 12-mode affine transformation of the AIR program for coregistration and eddy-current distortion correction. ${ }^{19}$ We then calculated the 6 elements of the diffusion tensor for each pixel by using multivariate linear fitting. ${ }^{13}$ After the diagonalization, 3 eigenvalues and eigenvectors were obtained. For the anisotropy map, FA was used. ${ }^{15}$

\section{Strategy for Delineation of Region of Interest}

The image analysis was based on manually delineated ROIs. To enhance reproducibility of the structure identification, we adopted a method aided by a brain normalization scheme (a "hybrid approach" method) in our previous publication. ${ }^{20}$ First, all brains were normalized to ICBM-DTI-81 atlas (http://www.loni.ucla.edu/Atlases/Atlas_ Detail.jsp?atlas_id = 15) by use of 12-mode affine transformation. To drive the registration, we used non-diffusion-weighted images. This adjusted the overall brain sizes and orientations across the subjects so that $2 \mathrm{D}$ observation sections with consistent orientations and locations could be extracted for the ROI drawing. We performed this initial brain registration using Landmarker (www.mristudio.org). ${ }^{20}$ After the registration, predefined $2 \mathrm{D}$ observation places (2 axial section at $z=82$ and 65 and 2 coronal sections at $y=89$ and 125 in the atlas space) were extracted. In this study, we placed multiple ROIs for large-fiber bundles. These multiple ROIs on the same white matter structures do not always lead to the same observations. The FA values of the same white matter tracts vary along their path. ${ }^{21}$ Individual axons may merge and exit a tract at many points. It is, therefore, anatomically possible that abnormal FAs are found only at a portion of white matter tracts. ${ }^{21}$

Fig 1 and the accompanying Table show all 45 ROIs placed at the specific anatomic locations. The shape of these ROIs followed the white matter parcellation map defined in the ICBM-DTI-81 atlas.

\section{Statistical Method}

The $t$ test and Mann-Whitney test were used for comparison of diffusion parameters between the patients and age-matched female control subjects. A $P$ value of .05 or less was considered statistically significant. To investigate the asymmetry between FA of the left and right hemispheres, we used 2 methods: 1) comparison of left and right FA values, and 2) LI for FA in all examined fiber tracts by using the formula LI $=($ left - right $) /[($ left + right $)]$. Significance of the hemispheric differences was assessed with the paired $t$ test for left-to-right comparison and the unpaired $t$ test for LI comparison. Bonferroni correction was applied. We performed all statistical analyses using STATA version 8.0 (StataCorp, College Station, Texas).

\section{Results}

A total of 32 patients with RTT and 37 control subjects were age matched when compared as 1 cohort $(P=.58)$ or in 3 subgroups: 1$)$ younger than 5 years $(2.8[\mathrm{SD}, 0.86]$ vs 2.7 [SD, $1.45] ; P=.9), 2)$ ages 5 to 10 years (7 [SD, 1.09] vs 7.6 [SD, $1.34] ; P=.414), 3)$ older than 10 years (13.5 [SD, 2.72] vs 12.8 $[\mathrm{SD}, 1.2] ; P=.472)$. Conventional MR imaging of patients and control subjects was obtained, and results were found to be unremarkable in control subjects. However, patients were noted to have microcephaly without other abnormalities. The Table shows the comparisons of FA between patients and control subjects. FA was not different in regions comprising the limbic system, namely the fornix and posterior cingulate gyrus. However, in the patients, FA for the anterior cingulum bundles (CG1) was significantly reduced. Of the cerebellar tracts, only the right middle cerebellar tract showed a trend of reduced FA. Significant reductions in FA were noted in the genu and splenium of the corpus callosum, and external capsule, with regional reductions in the anterior and posterior limbs of the internal capsule, posterior thalamic radiation, and frontal white matter in RTT. In addition, there was no difference in FA between patients with RTT and control subjects in 3 of the 4 ROIs in the posterior thalamic radiation with significantly higher FA in the posterior corona radiata of the patients (Table).

Patients were categorized by their capacity to speak: 1$)$ Mute $(n=13), 2)$ Single words $(n=13)$, and 3) Could speak in phrases or sentences $(n=6)$. As shown in Fig 2 , mean FA of the superior longitudinal fasciculus in control subjects and group 3 patients was not different $(0.38 \pm 0.04$ vs $0.375 \pm$ $0.02 ; t=0.615 ; P=.542)$. In a similar setting, there was no difference in the FA of the patients with RTT in group 1 and group 2 ( $0.32 \pm 0.05$ vs $0.33 \pm 0.05 ; t=-0.089 ; P=.929)$. But groups 1 and 2 with minimal speech were significantly different from group 3 and the control subjects (group 1 vs group 3: $t=-2.12 ; P=.046$; group 1 vs control: $t=3.77 ; P<$ .001 ; group 2 vs group $3: t=-2.03 ; P=.055$; group 2 vs 

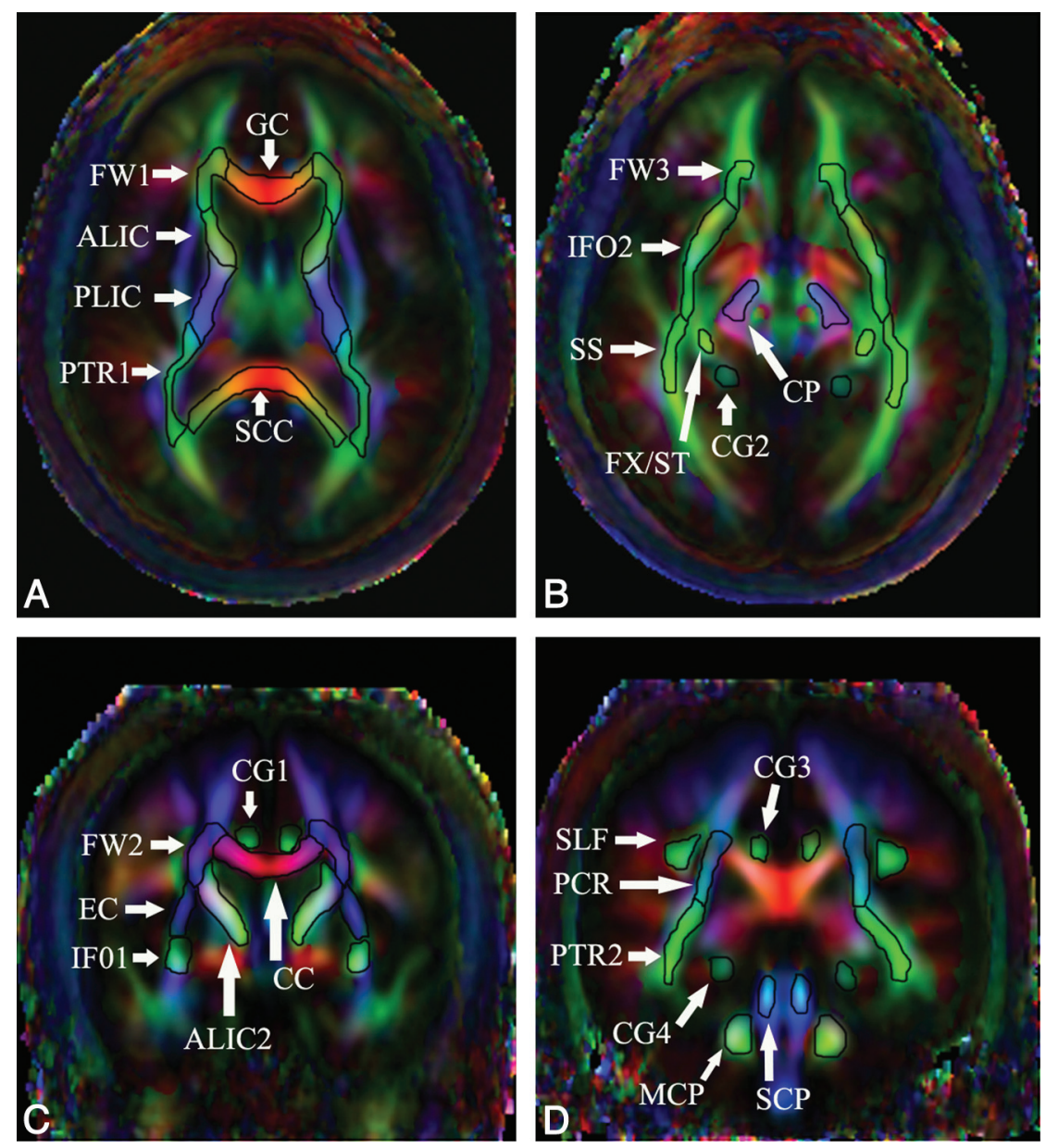

Fig 1. Total of 42 ROls (21 in each hemisphere) and 3 in the corpus callosum are shown.

control: $t=3.58 ; P<.001)$. This trend was not seen in any other region.

Our study did not find any correlation between FA values in the examined tracts and seizure status, head circumference, or walking ability. Hemispheric asymmetry analysis evaluating left/right differences showed that, in contrast to control subjects, asymmetry was not present in the inferior frontooccipital fasciculus $(P>.99)$, external capsule $(P>.99)$, superior longitudinal fasciculus $(P>.99)$, and posterior thalamic radiation $(P>.99)$ in patients with RTT. Additional LI analysis only showed a significant trend in the inferior frontooccipital fasciculus tracts $(P=.082)$ compared with control subjects. We noted hemispheric asymmetry in CG3 (posterior cingulate gyrus) of the patients $(P=.044)$ compared with control subjects, who showed no asymmetry $(P=.505)$.

\section{Discussion}

In the studied group of patients with RTT, the FA was reduced in the corpus callosum, internal capsule, and frontal white matter compared with control subjects. In contrast, mean FA in most of the regions comprising the limbic system and cerebellar tracts was similar in both groups. The posterior corona radiata was the only region in the patients with RTT where FA was greater than that of control subjects and this finding is in keeping with the observation in volumetric studies by Carter et al. ${ }^{7}$ Without histologic results, it is difficult to conclude about the exact mechanism of the FA decrease observed in this patient population. Past studies have suggested axonal involvement to explain FA reduction, ${ }^{20,22}$ but loss of myelin has not been shown to be a contributing factor. ${ }^{23}$ In patients with RTT, axonal vulnerability must be an important contributor to the reduced FA, as shown by reduced levels of $\mathrm{N}$-acetylaspartate in MR spectroscopic imaging. ${ }^{24}$

We noted 3 major correlations with clinical findings in RTT, the most significant of which was a correlation of FA in the superior longitudinal fasciculus with the ability to speak. The superior longitudinal fasciculus is one of the main association bundles that connect the external surface of temporoparieto-occipital regions with the convexity of the frontal lobe and, in part, is associated with phonologic speech. ${ }^{25,26}$ The left-more-than-right asymmetry in the superior longitudinal fasciculus, as seen in the control subjects, was not found in the patients.

Second, the presence of intact visual capabilities, which are used by some girls with RTT to communicate, may be in accordance with normal to increased FA in the posterior corona radiata. This observation was in contrast to the FA that was significantly reduced by greater margins in all regions of the corpus callosum affecting interhemispheric connectivity, probably because of immature neurons and their poor dendritic arborization. ${ }^{27}$ However, other white matter structures that are known to be associated with visual functions such as 


\begin{tabular}{|c|c|c|c|c|}
\hline \multicolumn{5}{|c|}{ Comparisons of FA between patients with RTT and control subjects } \\
\hline $\mathrm{ROI}$ & Side & RTT & Control & $P$ value \\
\hline$\overline{\mathrm{GC}}$ & & $0.555(0.052)$ & $0.67(0.033)$ & .0001 \\
\hline SCC & & $0.56(0.08)$ & $0.682(0.04)$ & .0001 \\
\hline CC & & $0.419(0.046)$ & $0.498(0.056)$ & .0001 \\
\hline \multirow[t]{2}{*}{ EC } & $\mathrm{R}$ & $0.291(0.059)$ & $0.321(0.037)$ & .008 \\
\hline & L & $0.296(0.042)$ & $0.337(0.038)$ & .0001 \\
\hline \multirow[t]{2}{*}{ IF01 } & $\mathrm{R}$ & $0.37(0.051)$ & $0.444(0.044)$ & .0001 \\
\hline & $\mathrm{L}$ & $0.374(0.051)$ & $0.427(0.045)$ & .0001 \\
\hline \multirow[t]{2}{*}{ IF02 } & $\mathrm{R}$ & 0.338 (0.039) & $0.392(0.051)$ & .0001 \\
\hline & L & $0.341(0.034)$ & $0.384(0.057)$ & .0018 \\
\hline \multirow[t]{2}{*}{ SS } & R & $0.403(0.047)$ & $0.433(0.043)$ & .034 \\
\hline & L & $0.392(0.044)$ & $0.435(0.046)$ & .001 \\
\hline \multirow[t]{2}{*}{ SLF } & R & $0.338(0.062)$ & $0.369(0.049)$ & .056 \\
\hline & $\mathrm{L}$ & $0.34(0.055)$ & $0.402(0.052)$ & .0001 \\
\hline \multirow[t]{2}{*}{ PCR } & R & $0.413(0.042)$ & $0.383(0.049)$ & .018 \\
\hline & L & $0.413(0.044)$ & $0.387(0.055)$ & .052 \\
\hline \multirow[t]{2}{*}{ ALIC } & R & $0.481(0.043)$ & $0.478(0.052)$ & $>.99$ \\
\hline & L & $0.473(0.049)$ & $0.482(0.046)$ & .92 \\
\hline \multirow[t]{2}{*}{ PLIC } & R & $0.547(0.033)$ & $0.565(0.024)$ & .02 \\
\hline & L & $0.548(0.037)$ & $0.566(0.029)$ & .16 \\
\hline \multirow[t]{2}{*}{ ALIC2 } & $\mathrm{R}$ & $0.475(0.051)$ & $0.509(0.042)$ & .012 \\
\hline & L & $0.463(0.047)$ & $0.51(0.042)$ & .0001 \\
\hline \multirow[t]{2}{*}{ PTR1 } & $\mathrm{R}$ & $0.428(0.046)$ & $0.455(0.04)$ & .018 \\
\hline & L & $0.424(0.051)$ & $0.439(0.045)$ & .346 \\
\hline \multirow[t]{2}{*}{ PTR2 } & R & $0.46(0.032)$ & $0.474(0.032)$ & .18 \\
\hline & L & $0.462(0.037)$ & 0.479 (0.038) & .12 \\
\hline \multirow[t]{2}{*}{ FW1 } & $\mathrm{R}$ & $0.356(0.059)$ & $0.394(0.052)$ & .014 \\
\hline & L & $0.352(0.046)$ & $0.401(0.051)$ & .0001 \\
\hline \multirow[t]{2}{*}{ FW2 } & R & $0.356(0.038)$ & $0.345(0.043)$ & .54 \\
\hline & $\mathrm{L}$ & $0.367(0.048)$ & $0.361(0.041)$ & $>.99$ \\
\hline \multirow[t]{2}{*}{ FW3 } & R & $0.394(0.047)$ & $0.455(0.05)$ & .0001 \\
\hline & L & $0.388(0.048)$ & $0.457(0.049)$ & .0001 \\
\hline \multirow[t]{2}{*}{$\mathrm{CP}$} & $\mathrm{R}$ & $0.552(0.06)$ & $0.557(0.058)$ & $>.99$ \\
\hline & L & $0.525(0.06)$ & $0.544(0.047)$ & .4 \\
\hline \multirow[t]{2}{*}{ SCP } & R & $0.542(0.098)$ & $0.567(0.084)$ & .7 \\
\hline & $\mathrm{L}$ & $0.537(0.091)$ & 0.568 (0.099) & .684 \\
\hline \multirow[t]{2}{*}{ МСР } & R & $0.518(0.053)$ & $0.55(0.046)$ & .078 \\
\hline & L & $0.531(0.059)$ & $0.535(0.057)$ & .914 \\
\hline \multirow[t]{2}{*}{ CG1 } & $\mathrm{R}$ & $0.275(0.056)$ & $0.336(0.045)$ & .0001 \\
\hline & L & $0.298(0.053)$ & $0.377(0.05)$ & .0001 \\
\hline \multirow[t]{2}{*}{ CG2 } & R & $0.301(0.059)$ & $0.31(0.055)$ & $>.99$ \\
\hline & $\mathrm{L}$ & $0.281(0.038)$ & $0.301(0.056)$ & .25 \\
\hline \multirow[t]{2}{*}{ CG3 } & R & $0.294(0.05)$ & $0.307(0.061)$ & .9 \\
\hline & L & $0.278(0.035)$ & $0.296(0.058)$ & .228 \\
\hline \multirow[t]{2}{*}{ CG4 } & $\mathrm{R}$ & $0.296(0.05)$ & $0.321(0.049)$ & .16 \\
\hline & L & $0.298(0.061)$ & $0.302(0.049)$ & $>.99$ \\
\hline FX/ST & $\mathrm{R}$ & $0.413(0.087)$ & $0.426(0.065)$ & $>.99$ \\
\hline & L & $0.418(0.074)$ & $0.442(0.063)$ & .334 \\
\hline
\end{tabular}

the sagittal stratum did not show increased FA. The increased FA of the posterior thalamic radiation, therefore, needs careful interpretation with increased numbers of patients. Study data also argue that, with few exceptions, white matter tracts in RTT are diffusely affected compared with control subjects. Except for the right middle cerebellar tract, other cerebellar tracts were generally spared. Right-to-left differences in some brain regions of patients with RTT suggest that neuronal connectivity may have become restricted before development of specific skills contributing to interhemispheric asymmetry in these patients.

The third significant observation is that of reduced FA in the anterior cingulate gyrus, which is particularly revealing in view of the characteristic mood and behavioral changes seen in patients with RTT. In a similar setting, changes in the anterior

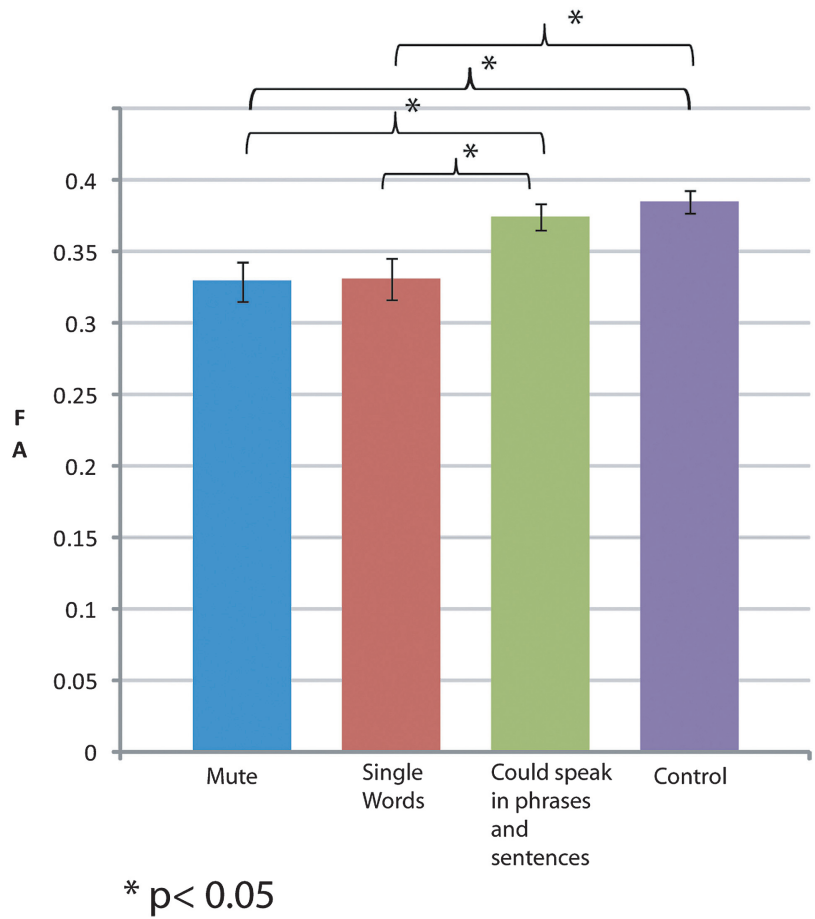

Fig 2. Comparison of FA among patients categorized by speech capacity in the superior longitudinal fasciculus and control subjects.

cingulate gyrus and other components of the limbic system are also reported in schizophrenic ${ }^{28}$ and autistic ${ }^{29}$ patients. In addition, the posterior cingulate gyrus showed hemispheric asymmetry in the patients, which was not present in the control subjects. Larger studies are required, however, to investigate the correlation between severe behavioral and emotional problems in patients with RTT and limbic system components.

Volumetric MR imaging studies in RTT have shown a significantly reduced cerebral volume affecting both gray and white matter. ${ }^{7,30,31}$ In patients with RTT, MR imaging spectroscopy has shown significant decreases in average concentrations of $\mathrm{N}$-acetylaspartate in both gray and white matter of the frontal and parietal lobes and insula compared with control subjects, demonstrating axonal involvement in RTT. ${ }^{24,23}$ Our in vivo observation confirms diffuse involvement of white matter tracts with areas of selective vulnerability in the limbic system components.

Longitudinal evaluations and studies in older subjects are needed for better understanding of changes in FA and its relationship to motor regression, and loss of speech noted in the older girls with RTT. Increased sample size will also allow better correlation of function to FA measurements. Improved technology with use of 3T or higher MR imaging modalities may provide better definition of tracts.

Clinical variability is currently assessed by neurologic examinations and mutation type. DTI studies would add specificity to these observations and help to understand the effects of individual mutations whose clinical effects are modified by age and X-inactivation in female patients. Therapeutic benefits may also be ascertained by their effects on neuronal tracts, either as improvement or lack of progressive reduction in FA. 


\section{Acknowledgments}

We thank Terri Brawner and Kathleen Kahl for their technical support in acquiring the MR imaging scans as well as Carolyn Gillen for nursing assistance during the MR imaging examinations.

\section{References}

1. Amir RE, Van den Veyver IB, Wan M, et al. Rett syndrome is caused by mutations in X-linked MECP2, encoding methyl-CpG-binding protein 2. Nature Genet 1999;23:185-88

2. Sirianni N, Naidu S, Pereira J, et al. Rett syndrome: confirmation of X-linked dominant inheritance, and localization of the gene to Xq28. Am J Human Genet 1998;63:1552-58

3. Kozinetz CA, Skender ML, MacNaughton N, et al. Epidemiology of Rett syndrome: a population-based registry. Pediatrics 1993;91:445-50

4. Hagberg B, Aicardi J, Dias K, et al. A progressive syndrome of autism, dementia, ataxia, and loss of purposeful hand use in girls: Rett's syndrome: report of 35 cases. Ann Neurol 1983;14:471-79

5. Naidu S. Rett syndrome: a disorder affecting early brain growth. Ann Neurol 1997;42:3-10

6. Armstrong D, Dunn JK, Antalffy B, et al. Selective dendritic alterations in the cortex of Rett syndrome. J Neuropathol Exp Neurol 1995;54:195-201

7. Carter JC, Lanham DC, Pham D, et al. Selective cerebral volume reduction in Rett syndrome: a multiple-approach MR imaging study. AJNR Am J Neuroradiol 2008;29:436-41

8. Kaufmann WE. Cortical development in Rett syndrome: molecular, neurochemical, and anatomic aspects. In: Kerr A, Engerstrom I, eds. Rett Disorder and the Developing Brain. Oxford, UK: Oxford University Press; 2001

9. Ronnett GV, Leopold D, Cai X, et al. Olfactory biopsies demonstrate a defect in neuronal development in Rett's syndrome. Ann Neurol 2003;54:206-18

10. Kishi N, Macklis JD. MECP2 is progressively expressed in post-migratory neurons and is involved in neuronal maturation rather than cell fate decisions. Mol Cell Neurosc 2004;27:306-21

11. Armstrong DD. Neuropathology of Rett syndrome. J Child Neurol 2005;20: 747-53

12. Bauman ML, Kemper TL, Arin DM. Pervasive neuroanatomic abnormalities of the brain in three cases of Rett's syndrome. Neurology 1995;45:1581-86

13. Basser PJ, Mattiello J, LeBihan D. Estimation of the effective self-diffusion tensor from the NMR spin echo. J Magn Reson B 1994;103:247-54

14. Mori S, Barker PB. Diffusion magnetic resonance imaging: its principle and applications. Anat Rec 1999;257:102-09
15. Pierpaoli C, Basser PJ. Toward a quantitative assessment of diffusion anisotropy. Magn Reson Med 1996;36:893-906

16. Pruessmann KP, Weiger M, Scheidegger MB, et al. SENSE: sensitivity encoding for fast MRI. Magn Reson Med 1999;42:952-62

17. Jones DK, Horsfield MA, Simmons A. Optimal strategies for measuring diffusion in anisotropic systems by magnetic resonance imaging. Magn Reson Med 1999;42:515-25

18. Jiang H, van Zijl PC, J K, et al. DTIStudio: resource program for diffusion tensor computation and fiber bundle tracking. Comput Methods Programs Biomed 2006;81:106-16

19. Woods RP, Grafton ST, Holmes CJ, et al. Automated image registration: I. General methods and intrasubject, intramodality validation. J Comput Assist Tomogr 1998;22:139-52

20. Douaud G, Behrens TE, Poupon $\mathrm{C}$, et al. In vivo evidence for the selective subcortical degeneration in Huntington's disease. Neuroimage 2009;46: 958-66

21. Stieltjes B, Kaufmann WE, van Zijl PC, et al. Diffusion tensor imaging and axonal tracking in the human brainstem. Neuroimage 2001;14:723-35

22. Beaulieu C, Allen PS. Determinants of anisotropic water diffusion in nerves. Magn Reson Med 1994;31:394-400

23. Song SK, Sun SW, Ramsbottom MJ, et al. Dysmyelination revealed through MRI as increased radial (but unchanged axial) diffusion of water. Neuroimage 2002;17:1429-36

24. Horska A, Naidu S, Herskovits EH, et al. Quantitative 1H MR spectroscopic imaging in early Rett syndrome. Neurology 2000;54:715-22

25. Tanabe H, Sawada $\mathrm{T}$, Inoue N, et al. Conduction aphasia and arcuate fasciculus. Acta Neurol Scand 1987;76:422-27

26. Boatman D, Gordon B, Hart J, et al. Transcortical sensory aphasia: revisited and revised. Brain 2000;123(Pt 8):1634-42

27. Belichenko PV, Wright EE, Belichenko NP, et al. Widespread changes in dendritic and axonal morphology in Mecp2-mutant mouse models of Rett syndrome: evidence for disruption of neuronal networks. J Comp Neurol 2009;514:240-58

28. White T, Cullen K, Rohrer LM, et al. Limbic structures and networks in children and adolescents with schizophrenia. Schizophr Bull 2008;34:18-29

29. Craig MC, Zaman SH, Daly EM, et al. Women with autistic-spectrum disorder: magnetic resonance imaging study of brain anatomy. Br J Psychiatry 2007;191: 224-28

30. Reiss AL, Faruque F, Naidu S, et al. Neuroanatomy of Rett syndrome: a volumetric imaging study. Ann Neurol 1993;34:227-34

31. Naidu S, Kaufmann WE, Abrams MT, et al. Neuroimaging studies in Rett syndrome. Brain Dev 2001;23 Suppl 1:S62-71 